\title{
Altered baseline brain activity in children with bipolar disorder during mania state: a resting-state study
}

This article was published in the following Dove Press journal:

Neuropsychiatric Disease and Treatment

17 February 2014

Number of times this article has been viewed

\section{Dali Lu' \\ Qing Jiao \\ Yuan Zhong ${ }^{3,4}$ \\ Weijia Gao' \\ Qian Xiao' \\ Xiaoqun Liu' \\ Xiaoling $\operatorname{Lin}^{5}$ \\ Wentao Cheng ${ }^{6}$ \\ Lanzhu Luo ${ }^{6}$ \\ Chuanjian $X^{3}$ \\ Guangming $\mathrm{Lu}^{2}$ \\ Linyan Su'}

'Mental Health Institute of the Second Xiangya Hospital, Key Laboratory of Psychiatry and Mental Health of Hunan Province, Central South University, Changsha, People's Republic of China; ${ }^{2}$ Department of Radiology, Taishan Medical University, Taian, People's Republic of China; ${ }^{3}$ Department of Medical Imaging, Jinling Hospital, Nanjing University School of Medicine, Nanjing, People's Republic of China; ${ }^{4}$ School of Psychology, Nanjing Normal University, Nanjing, People's Republic of China; ${ }^{5}$ School of Nursing of Central South University, Changsha, People's Republic of China; ${ }^{6}$ Department of Pediatric and Geriatric Psychiatry, Fuzhou Neuropsychiatric Hospital, Fuzhou, People's Republic of China

Correspondence: Linyan Su Mental Health Institute of the Second Xiangya Hospital, Key Laboratory of Psychiatry and Mental Health of Hunan Province, Central South University, 139 Renmin Middle Road, Changsha, 4I 00 I I, People's Republic of China

$\mathrm{Tel} / \mathrm{Fax}+8673 \mathrm{I} 85292474$

Email childpsy2012@I63.com
Background: Previous functional magnetic resonance imaging (fMRI) studies have shown abnormal functional connectivity in regions involved in emotion processing and regulation in pediatric bipolar disorder (PBD). Recent studies indicate, however, that task-dependent neural changes only represent a small fraction of the brain's total activity. How the brain allocates the majority of its resources at resting state is still unknown. We used the amplitude of lowfrequency fluctuation (ALFF) method of fMRI to explore the spontaneous neuronal activity in resting state in PBD patients.

Methods: Eighteen PBD patients during the mania phase and 18 sex-, age- and educationmatched healthy subjects were enrolled in this study and all patients underwent fMRI scanning. The ALFF method was used to compare the resting-state spontaneous neuronal activity between groups. Correlation analysis was performed between the ALFF values and Young Mania Rating Scale scores.

Results: Compared with healthy controls, PBD patients presented increased ALFF in bilateral caudate and left pallidum as well as decreased ALFF in left precuneus, left superior parietal lobule, and bilateral inferior occipital gyrus. Additionally, ALFF values in left pallidum were positively correlated with Young Mania Rating Scale score in PBD.

Conclusion: The abnormal resting-state neuronal activities of the basal ganglia, parietal cortex, and occipital cortex may play an important role in the pathophysiology in PBD patients.

Keywords: resting-state fMRI, amplitude of low-frequency fluctuation, child, bipolar disorder

\section{Introduction}

Childhood-onset bipolar disorder (BD) has been shown to be associated with shorter periods of euthymia, higher rates of psychosis, more recurrences, and greater likelihood of suicide attempts and violence than adult-onset BD. ${ }^{1,2}$ A follow-up study involving 24-year olds noted that pediatric BD (PBD) patients or subsyndromal youth BD patients showed significant impairment in psychosocial functioning and had higher mental health treatment utilization. ${ }^{3}$ Childhood-onset $\mathrm{BD}$ cases are more treatment resistant and have a worse prognosis than adult-onset $\mathrm{BD},{ }^{4}$ which poses a great treatment challenge for clinicians and a heavy burden on affected families. Thus, there is a pressing need for greater neurobiological understanding of PBD.

Toward that end, structural magnetic resonance imaging (MRI) studies have reported abnormalities in affect-processing regions, including abnormalities in prefrontal cortex, amygdale, superior temporal gyrus, and hippocampal volumes in PBD. ${ }^{5,6}$ Other studies have shown that abnormalities converge in the prefrontal white matter and, 
in particular, prefrontal and temporal associative tracts are known to be involved in emotion in PBD. ${ }^{7,8}$

Most functional MRI (fMRI) studies concerning PBD have also found abnormal affect-processing circuitry, which may cause emotional dysregulation and sociocognitive difficulties. Previous studies have shown reduced activation of right rostral ventrolateral prefrontal cortex together with increased activity in anterior cingulate, amygdala, and paralimbic cortex in PBD patients compared to healthy controls using a variety of cognitive tasks. ${ }^{9}, 10$ Task-related changes in neural activation, however, only represent a small fraction (perhaps $<5 \%$ ) of the brain's total activity. ${ }^{11,12}$ Intrinsic activity consumes more of the brain's energy than external stimuli. ${ }^{13}$ Thus, knowing to what the brain allocates the majority of its resources is essential for understanding neural mechanisms associated with PBD, but this is something that remains unclear in the present day.

Resting-state intrinsic activity may be measured by the amplitude of low-frequency fluctuations (ALFF) of the blood oxygenation level-dependent (BOLD) fMRI signal. ${ }^{14,15}$ The ALFF approach may reflect spontaneous neuronal activity and physiological states in the region and help to determine abnormal activity within the entire brain. ${ }^{16}$ That is to say, this method could be a more direct index reflecting the amplitude of regional spontaneous neuronal activity and can be used to locate specific, impaired brain regions in the resting state, as has been demonstrated by Zang et al. ${ }^{17}$ Another advantage of the ALFF approach is that ALFF may help to avoid potential bias induced by the selection of the "seed" voxels in functional connectivity analysis. Indeed, since Zang et al ${ }^{17}$ demonstrated, ALFF has been used in many psychiatric studies to reveal regional brain activity alteration. ${ }^{17-19}$

In this study, we first adopted the ALFF method to examine the hypothesis that activity alterations occur in affectprocessing regions in $\mathrm{PBD}$ patients in resting state, then the ALFF values of the brain regions that showed significant difference between the patients and controls were correlated with the Young Mania Rating Scale (YMRS) scores.

\section{Methods}

\section{Subjects}

A total of 36 right-handed, 12-18-year-old subjects were recruited, including $18 \mathrm{PBD}$ patients and 18 normal controls. Patients were recruited at the Mental Health Institute of the Second Xiangya Hospital, Changsha, People's Republic of China. Healthy controls were recruited from a local middle school. The study protocol was approved by the ethics committee of the Second Xiangya Hospital. Oral and written informed consent was obtained from parents and children. The PBD $(\mathrm{n}=18)$ inclusion criteria were meeting Diagnostic and Statistical Manual of Mental Disorders, Fourth Edition Text Revision (DSM-IV-TR) criteria for BD, including at least one episode meeting full DSM-IV-TR criteria for hypomania ( $\geq 4$ days) or mania ( $\geq 7$ days). ${ }^{20}$ All participants were evaluated by two qualified psychiatrists using the Schedule for Affective Disorders and Schizophrenia for School-Age Children-Present and Lifetime Version (K-SADS-PL), and the Wechsler Abbreviated Scale of Intelligence for Chinese Revised (WASI-CR) was administered as an overall measure of their cognitive ability. ${ }^{21}$ All participants also completed the Young Mania Rating Scale (YMRS) and Mood and Feelings Questionnaire (MFQ) to evaluate mood of the patient within 24 hours of scanning. ${ }^{22}$ According to Wood et a ${ }^{122}$ and Rich et al, ${ }^{23}$ manic state should be YMRS $>26$ and MFQ $<18$; hypomanic state should be YMRS $>12$ but $<18$ and MFQ $<18$.

Exclusion criteria were left-handedness, IQ $<80$, pervasive developmental disorder, neurological illness, metal implants, history of skull fracture or head trauma with loss of consciousness longer than 5 minutes, and substance abuse.

\section{Data acquisition}

Functional imaging was performed on a 3.0 T Siemens Trio scanner (Siemens, Munich, Germany). During the resting-state fMRI scanning, subjects were asked to lie supine with their heads kept still and to keep their eyes closed without thinking anything systematically nor falling asleep. Resting-state functional images were acquired with 33 axial slices, repetition time 2,000 milliseconds, echo time 30 milliseconds, slice thickness $4.0 \mathrm{~mm}$, gap $0.4 \mathrm{~mm}$, flip angle $90^{\circ}$, field of view $240 \mathrm{~mm} \times 240 \mathrm{~mm}$, and matrix $64 \times 64$, lasting for 8 minutes. Structural images were acquired by using threedimensional magnetization-prepared rapid gradient-echo sequence (repetition time 2,300 milliseconds; echo time 2.98 milliseconds; inversion time 900 milliseconds; field of view $256 \mathrm{~mm} \times 256 \mathrm{~mm}$; flip angle $90^{\circ}$; matrix $256 \times 256$ ).

\section{Image analysis}

Spatial preprocessing of fMRI was conducted using the Statistical Parametric Mapping package (v 8; Wellcome Trust Centre for Neuroimaging, London, UK). For the MRI signal to reach a steady state and the subjects to get used to the scanner noise, we discarded the first 10 volumes of the resting-state images. Then, the remaining images were preprocessed by performing the following steps: slice timing; realignment; spatial normalization to the standard Montreal Neurological Institute echo-planar imaging template in the Statistical 
Parametric Mapping package; and resampling to $3 \mathrm{~mm}$ cubic voxels, followed by spatial smoothing with $4 \mathrm{~mm}$ full-width at half-maximum Gaussian kernel. Participants were excluded if their head motion was $>1.5 \mathrm{~mm}$ maximum or $1.5^{\circ}$ of any angular motion throughout the course of the scan. ${ }^{24}$

\section{ALFF analysis}

The ALFF was performed using the Resting-State fMRI Data Analysis Toolkit (version 1.8; The State Key Laboratory of Cognitive Neuroscience and Learning, Beijing Normal University, Beijing, People's Republic of China). The time series for each voxel was filtered (band-pass filtering: 0.01-0.08 Hz) to remove the effect of low-frequency drifts and high-frequency noise. Then, the filtered time series was transformed to a frequency domain using a fast Fourier transform (FFT) (parameters: taper percent 0 , FFT length shortest) and the power spectrum was then obtained by square-rooted FFT and averaged across $0.01-0.08 \mathrm{~Hz}$ at each voxel. This averaged square root was taken as the ALFF. Then, a mask made by the Montreal Neurological Institute template to ensure matching with the normalization step was used to remove the tissues outside the brain. For standardization purposes, the ALFF of each voxel was divided by the global mean ALFF value to standardize data across subjects. ${ }^{17}$

\section{Correlation analysis}

Brain regions showing significant ALFF alteration between the PBD patients and healthy controls were treated as the regions of interest (ROIs). The ALFF values of the voxels in the ROIs were averaged to present the ALFF of the ROIs. Pearson's correlation analysis was performed between the ALFF values of the ROIs and the YMRS scores of the patients to evaluate the relation between the ALFF of a brain region and manic severity.

\section{Statistical analysis}

Two-sample $t$-test was applied to compare the results between PBD patients and healthy controls. Monte Carlo simulation was utilized for the correction for multiple comparisons using the Resting-State fMRI Data Analysis Toolkit (version 1.8). A corrected significance level of $P<0.05$ was obtained by a combined threshold of $P<0.001$ for each voxel and an extent threshold of $>6$ voxels. The independent-sample $t$-test was used to compare the demographic data using SPSS software (v 11.5; IBM Corporation, Armonk, NY, USA).

\section{Results}

\section{Subjects}

PBD patients and healthy subjects completed the whole study and did not show any statistical differences in age, sex, or Full-Scale IQ. The demographic and clinical data are presented in Table 1. No subject had fallen asleep nor was excluded due to head motion greater than $1.5 \mathrm{~mm}$ or any angular motion greater than $1.5^{\circ}$ during scanning.

\section{ALFF: PBD patients versus control subjects}

As shown in Table 2 and Figure 1, compared to the controls, the PBD group showed significant ALFF decrease in left precuneus, left superior parietal lobule, and bilateral inferior occipital gyrus, and increase in the bilateral caudate and left pallidum.

\section{Correlations between ALFF values and YMRS scores}

As shown in Table 3 and Figure 2, significant positive correlation was found between the ALFF value of the left pallidum $(r=0.518, P=0.028)$ and the YMRS score in the PBD group.

Table I Participant demographic data in PBD $(n=18)$ versus typically HC ( $n=18)$ subjects

\begin{tabular}{|c|c|c|c|c|}
\hline Characteristics & PBD & HC & $t / \chi^{2}$ & $\boldsymbol{P}$ \\
\hline Age, years & $|5| \pm.|.8|$ & $|4.13 \pm 1.6|$ & 1.75 & 0.09 \\
\hline Full-Scale IQ & $98.5 \pm|3.5|$ & $105.00 \pm 7.72$ & -1.87 & 0.07 \\
\hline Education (years) & $8.17 \pm 1.69$ & $7.44 \pm 2.28$ & 1.08 & 0.29 \\
\hline Sex (male/female), $n$ & $6 / 12$ & $6 / 12$ & 0 & I \\
\hline YMRS scores & $33.40 \pm 6.09$ & $3.67 \pm 2.11$ & 19.56 & 0.00 \\
\hline MFQ scores & $6.89 \pm 2.59$ & $6.39 \pm 3.36$ & 0.50 & 0.62 \\
\hline \multicolumn{5}{|c|}{ K-SADS-PL current comorbidity diagnoses, n (\%) } \\
\hline ADHD & I (5.56) & & & \\
\hline OCD & $2(I 1.12)$ & & & \\
\hline Anxiety & I (5.56) & & & \\
\hline \multicolumn{5}{|l|}{ Medications, n (\%) } \\
\hline Lithium & 7 (38.9) & & & \\
\hline Valproate & $8(44.4)$ & & & \\
\hline Atypical antipsychotics & $13(72.2)$ & & & \\
\hline Antidepressants & $3(16.7)$ & & & \\
\hline None & $2(I I . I)$ & & & \\
\hline Onset age, years & $13.6 \pm 1.7$ & & & \\
\hline Illness duration, months & $15.6 \pm 13.0$ & & & \\
\hline $\begin{array}{l}\text { Family BD history } \\
\text { (yes/no), } \mathrm{n}\end{array}$ & $5 / 13$ & & & \\
\hline $\begin{array}{l}\text { Psychotic symptoms } \\
\text { (yes/no), n }\end{array}$ & $9 / 9$ & & & \\
\hline BP-I/BP-II, n & $14 / 4$ & & & \\
\hline \multicolumn{5}{|c|}{$\begin{array}{l}\text { Notes: All patients were of Han Chinese ethnicity/race. Data are presented as } \\
\text { mean } \pm \text { standard deviation, except where indicated otherwise. }\end{array}$} \\
\hline \multicolumn{5}{|c|}{$\begin{array}{l}\text { Abbreviations: PBD, pediatric bipolar disorder; HC, healthy control; IQ, intelligence } \\
\text { quotient; YMRS, Young Mania Rating Scale; MFQ, Mood and Feelings Questionnaire; } \\
\text { K-SADS-PL, Schedule for Affective Disorders and Schizophrenia for School-Age } \\
\text { Children-Present and Lifetime Version; ADHD, attention-deficit/hyperactivity } \\
\text { disorder; OCD, obsessive-compulsive disorder; BD, bipolar disorder; BP-I, bipolar } \\
\text { disorder type I; BP-II, bipolar disorder type II. }\end{array}$} \\
\hline
\end{tabular}


Table 2 Brain regions showing increased/decreased ALFF in PBD versus $\mathrm{HC}$ subjects

\begin{tabular}{|c|c|c|c|c|c|c|c|}
\hline \multirow{2}{*}{$\begin{array}{l}\text { Brain regions } \\
\text { of ALFF } \\
\text { PBD vs HC } \\
\end{array}$} & \multirow[t]{2}{*}{ Side } & \multirow[t]{2}{*}{ BA } & \multicolumn{3}{|c|}{$\begin{array}{l}\text { Talairach } \\
\text { coordinates (MNI) }\end{array}$} & \multirow[t]{2}{*}{$t$-statistic } & \multirow[t]{2}{*}{$K$} \\
\hline & & & $\bar{x}$ & $\mathbf{Y}$ & $\mathbf{Z}$ & & \\
\hline \multicolumn{8}{|l|}{$\mathrm{PBD}>\mathrm{HC}$} \\
\hline Caudate & Left & & -15.00 & 6.00 & 15.00 & 4.18 & 42 \\
\hline Caudate & Right & & 12.00 & 6.00 & 15.00 & 4.05 & 55 \\
\hline Pallidum & Left & & -15.00 & 6.00 & -3.00 & 3.52 & 10 \\
\hline \multicolumn{8}{|l|}{$\mathrm{PBD}<\mathrm{HC}$} \\
\hline Precuneus & Left & 7 & -18.00 & -63.00 & 57.00 & -5.78 & 10 \\
\hline $\begin{array}{l}\text { Superior } \\
\text { parietal lobule }\end{array}$ & Left & 7 & -24.00 & -69.00 & 45.00 & -4.02 & 37 \\
\hline $\begin{array}{l}\text { Inferior } \\
\text { occipital gyrus }\end{array}$ & Left & 18 & -30.00 & -87.00 & -15.00 & -3.55 & 18 \\
\hline $\begin{array}{l}\text { Inferior } \\
\text { occipital gyrus }\end{array}$ & Right & 18 & 42.00 & -84.00 & -15.00 & -3.35 & 13 \\
\hline
\end{tabular}

Abbreviations: ALFF, amplitude of low-frequency fluctuation; BA, Brodmann area; $\mathrm{HC}$, healthy controls; $\mathrm{PBD}$, pediatric bipolar disorder; $\mathrm{K}$, cluster size in $3 \times 3 \times 3 \mathrm{~mm}^{3}$ voxels; MNI, Montreal Neurological Institute.

\section{Discussion}

To the best of our knowledge, this is the first resting-state fMRI study to examine PBD during mania state using the ALFF method. In order to advance early detection and effective intervention, it is important for us to have a good understanding of the early natural history of BD. In fact, childhood and adolescence are crucial developmental periods. Frontal and parietal lobes attain an adult level of development at around 12 years of age and the temporal lobe at around 16 years, whereas cortical white matter refinements continue until near age $20 .{ }^{25}$ In addition, PBD that is less influenced by psychological and social factors may represent a more homogeneous subtype of BD than adult-onset BD in genetic studies. That is to say, PBD may be closer in nature to BD disease. Only three neuroimaging studies have evaluated PBD using resting-state fMRI method. ${ }^{26-28}$ All of these studies reported abnormalities in affect circuits, such as abnormal activities in prefrontal cortex ${ }^{26,27}$ and superior temporal gyrus ${ }^{28,29}$ and abnormalities in cognitive circuits, such as abnormal activities in anterior cingulate $\operatorname{cortex}^{28,29}$ and caudate. ${ }^{26,27}$

Two key findings are present in our study. First, the PBD group displayed significant ALFF increase in the bilateral caudate and the left pallidum, and there was a strong positive association between the ALFF value of left pallidum and YMRS score. Caudate nucleus and pallidum are major components of the basal ganglia. Investigators have reported increased activity in the caudate both in adult $\mathrm{BD}$ and childhood $\mathrm{BD}$ during mania state. ${ }^{29-32} \mathrm{~A}$ task-independent spontaneous resting-state functional connectivity (RSFC) study in PBD involved youth BD patients ( $\mathrm{n}=15)$ at euthymic state, showing decreased resting-state functional connectivity between the right superior temporal gyrus and left caudate. ${ }^{26}$ The abnormal neuronal activity of the caudate shown in our findings may contribute to the abnormal connectivity between the two regions. Thus, our work is an important first step toward locating specific, impaired brain regions intrinsic to PBD in the resting state. Previous structural MRI studies have shown that adult $\mathrm{BD}$ and $\mathrm{PBD}$ are characteristic with increased volume of the pallidum. ${ }^{33,34}$ One fMRI study also showed increased activity in left pallidum in BD during mania state compared to healthy subjects. ${ }^{35}$ Our findings are consistent with the abovementioned study. The pallidum is involved in the regulation of voluntary movement. If this brain region is damaged, movement disorders can result. This may help to explain the symptom of increased motor activity

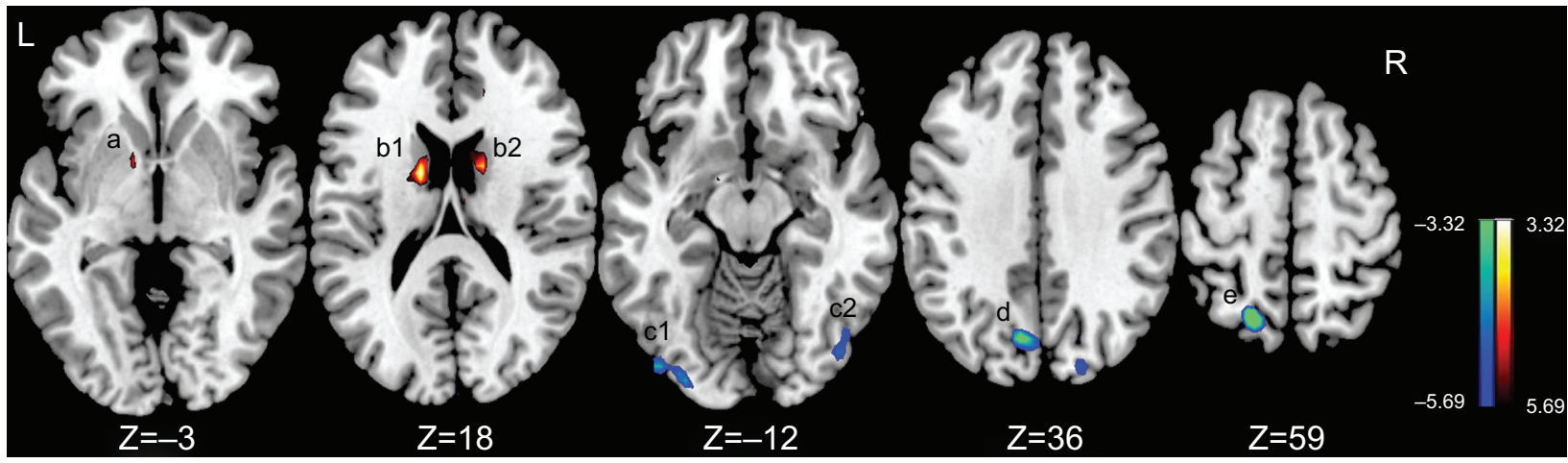

Figure I Brain regions with increased/decreased amplitude of low-frequency fluctuation (ALFF) in pediatric bipolar disorder patients are superimposed on a TI template. Notes: The ALFF showed a decrease (cold colors) in the left precuneus (d), left superior parietal lobule (e), and bilateral inferior occipital gyrus (cl and c2), and an increase (hot colors) in bilateral caudate (bl and b2) and left pallidum (a). The voxels with $P=0.00 \mathrm{I}$ and a cluster size of 6 were used to identify the clusters with significant differences between the pediatric bipolar disorder and healthy control groups $(P<0.05$, corrected $)$. Patients versus controls; two-sample $t$-test; $P<0.05$, corrected.

Abbreviations: L, left; R, Right. 
Table 3 Brain regions with significant between-group differences in ALFF and YMRS

\begin{tabular}{lll}
\hline Brain region & Side & YMRS $(r, P)$ \\
\hline Caudate & Left & $0.40,0.10$ \\
Caudate & Right & $0.11,0.69$ \\
Pallidum & Left & $0.52,0.03^{*}$ \\
Precuneus & Left & $0.17,0.51$ \\
Superior parietal lobule & Left & $-0.06,0.82$ \\
Inferior occipital gyrus & Left & $0.02,0.93$ \\
Inferior occipital gyrus & Right & $0.01,0.96$ \\
\hline
\end{tabular}

Note: $* P<0.05$.

Abbreviations: ALFF, amplitude of low-frequency fluctuation; YMRS, Young Mania Rating Scale.

energy during mania state. Above all, PBD was characterized in the present study by increased activity in the basal ganglia, including caudate and pallidum, consistent with previous studies. $^{29-34}$

The second key finding of the present study was a significantly decreased ALFF in the left precuneus, left superior parietal lobule, and bilateral inferior occipital gyrus of the PBD patients compared with that of the controls. The precuneus, a part of the superior parietal lobule, has been considered to be the core node of the default mode network that is activated during resting consciousness in which people do not engage intentionally in sensory or motor activity. ${ }^{36,37}$ In addition, precuneus and task superior parietal lobule are also involved in various executive functions, including planning, switching, and working memory. ${ }^{38,39}$ Investigators have reported decreased activity in precuneus and superior parietal lobule using emotionally valenced face tasks. ${ }^{23,40} \mathrm{~A}$ significant functional aspect of inferior occipital gyrus is that it contains the primary visual cortex. Occipital lesions may cause visual hallucinations. This may help to explain why hallucination symptoms frequently occur in pediatric mania. Further study is needed to explore this phenomenon more fully, however.

A hypothetical parietal-basal ganglia or occipital-basal ganglia model instead of frontostriatal circuitry used in previous studies may help to explain the imbalance of brain activity between parietal cortex, ${ }^{41,42}$ occipital cortex, and basal ganglia systems in the PBD group compared to the control group in our findings. The decreased neural activity of parietal cortex and occipital cortex may reflect that a smaller cognitive effort is required to exert effective excitatory control over basal ganglia regions in PBD. That is to say, the caudate nucleus receives inputs from the parietal and occipital cortices and sends stimulated information to the pallidum, which then excites the basal ganglia. The basal ganglia is associated with a variety of functions, including action selection, action gating, reward-based learning, motor preparation, and cognitive

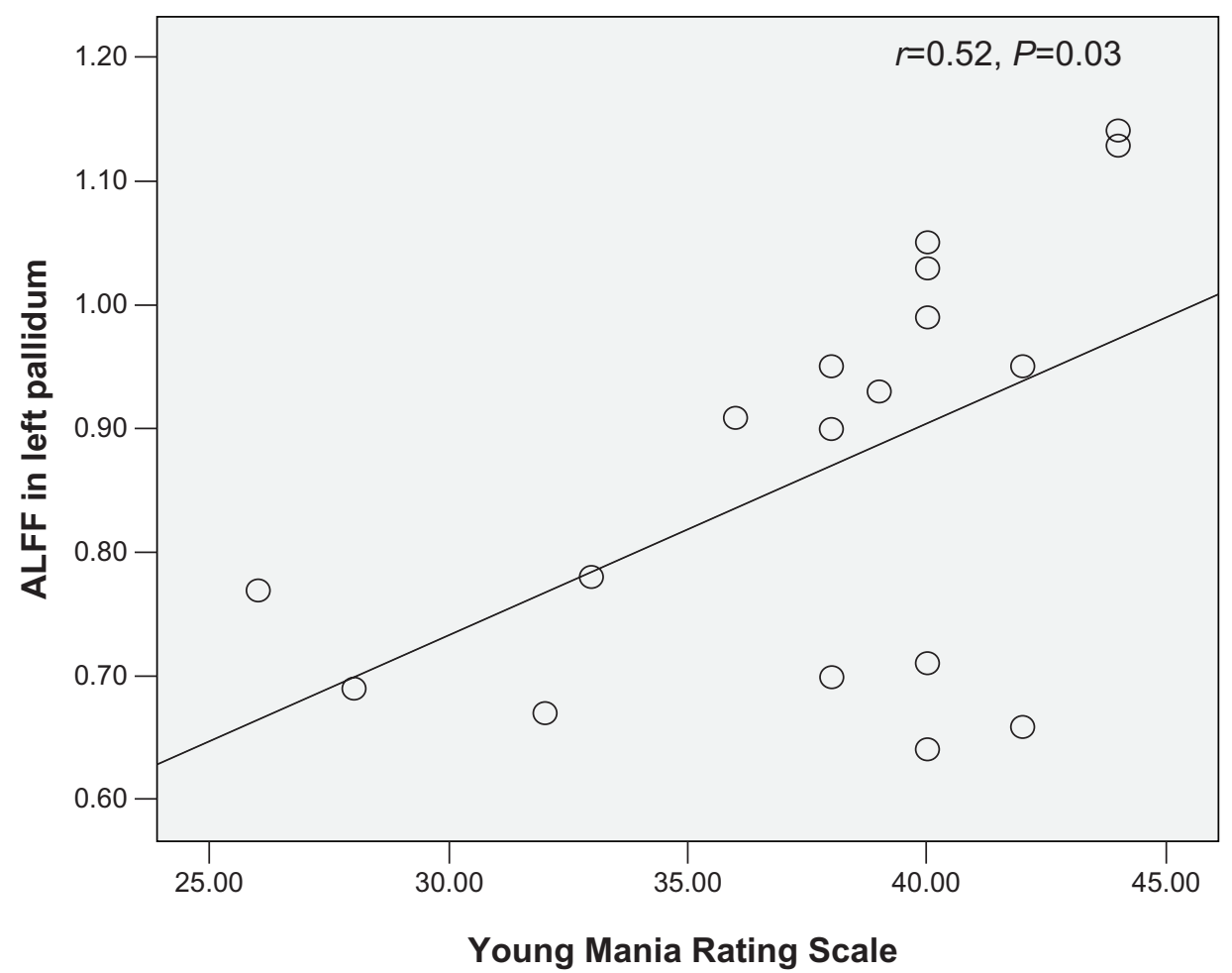

Figure 2 Scatter plots showing significant positive correlation between total Young Mania Rating Scale scores and regional amplitude of low-frequency fluctuation (ALFF) values in the left pallidum in pediatric bipolar disorder patients $(P<0.05)$. 
and emotional functions. ${ }^{43,44}$ Thus, the increased basal ganglia excitation in the resting state may underlie the behavioral abnormalities observed in mania, such as elevated selfesteem, being more talkative than usual, flight of thought, a reduced need for sleep, easily distracted and so on.

\section{Limitations}

There are some limitations in our study including comorbidity, psychotropic medications, and sample size. First, our study was a cross-sectional study, so it may be fruitful to investigate different episodes in the same subjects, such as depressive state or euthymic state. Second, the pharmaceutical effect is not excluded in our study, and this may be a confounding factor in interpreting the results, although recent BD research suggests that such psychotropic medications have demonstrated no significant effect on the BOLD signal in fMRI studies. ${ }^{45}$ Third, the sample size in our study was small, and our findings need to be further verified with a larger sample.

\section{Conclusion}

In all, the regions that showed significant ALFF change in the PBD mania group were parts of the basal ganglia and parietal cortex and the occipital cortex system, which are important for affect-processing, decision-making, and social cognition. ${ }^{46,47}$ These findings support our hypothesis that the abnormality in resting-state activity of PBD mania patients is mainly located in the basal ganglia and parietal and occipital systems. Additionally, the ALFF approach may help with locating impaired regions in $\mathrm{PBD}$ patients in functional brain research, consistent with previous studies on other mental disorders..$^{17,18}$

\section{Author contributions}

Dr Linyan Su designed the study. Dali Lu, Qian Xiao, Weijia Gao, Xiaoqun Liu, Xiaoling Lin, Wentao Cheng, and Lanzhu Luo collected the original imaging data. Qing Jiao, Yuan Zhong, Chuanjian Xu, and Guangming Lu managed and analyzed the imaging data, and Dali Lu wrote the first draft of the manuscript. All authors contributed to and approved the final manuscript.

\section{Acknowledgments}

This study was supported by grants from the National Natural Science Foundation of China (No 81171291 and 81201077) and Key Program for Guangming Lu (grant No BWS11J063 and 10z026) and Humanity and Social Science Youth foundation of Ministry of Education (No 11YJC190039).

\section{Disclosure}

The authors report no conflicts of interest in this work.

\section{References}

1. Perlis RH, Miyahara S, Marangell LB, et al; STEP-BD Investigators. Long-term implications of early onset in bipolar disorder: data from the first 1000 participants in the systematic treatment enhancement program for bipolar disorder (STEP-BD). Biol Psychiatry. 2004;55(9):875-881.

2. Schulze TG, Müller DJ, Krauss H, et al. Further evidence for age of onset being an indicator for severity in bipolar disorder. JAffect Disord. 2002;68(2-3):343-345.

3. Lewinsohn PM, Klein DN, Seeley JR. Bipolar disorder during adolescence and young adulthood in a community sample. Bipolar Disord. 2000;2(3 Pt 2):281-293.

4. Geller B, Bolhofner K, Craney JL, Williams M, DelBello MP, Gundersen K. Psychosocial functioning in a prepubertal and early adolescent bipolar disorder phenotype. $J$ Am Acad Child Adolesc Psychiatry. 2000;39(12):1543-1548.

5. Frazier JA, Breeze JL, Makris N, et al. Cortical gray matter differences identified by structural magnetic resonance imaging in pediatric bipolar disorder. Bipolar Disord. 2005;7(6):555-569.

6. Kalmar JH, Wang F, Chepenik LG, et al. Relation between amygdala structure and function in adolescents with bipolar disorder. $\mathrm{Am}$ Acad Child Adolesc Psychiatry. 2009;48(6):636-642.

7. Mahon K, Burdick KE, Szeszko PR. A role for white matter abnormalities in the pathophysiology of bipolar disorder. Neurosci Biobehav Rev. 2010;34(4):533-554.

8. Versace A, Ladouceur CD, Romero S, et al. Altered development of white matter in youth at high familial risk for bipolar disorder: a diffusion tensor imaging study. J Am Acad Child Adolesc Psychiatry. 2010;49(12):1249-1259.

9. Pavuluri MN, O'Connor mm, Harral E, Sweeney JA. Affective neural circuitry during facial emotion processing in pediatric bipolar disorder. Biol Psychiatry. 2007;62(2):158-167.

10. Pavuluri MN, Passarotti AM, Harral EM, Sweeney JA. An fMRI study of the neural correlates of incidental versus directed emotion processing in pediatric bipolar disorder. J Am Acad Child Adolesc Psychiatry. 2009;48(3):308-319.

11. Fox MD, Raichle ME. Spontaneous fluctuations in brain activity observed with functional magnetic resonance imaging. Nat Rev Neurosci. 2007;8(9):700-711.

12. Raichle ME. Neuroscience. The brain's dark energy. Science. 2006; 314(5803):1249-1250.

13. Raichle ME, Mintun MA. Brain work and brain imaging. Аnпи Rev Neurosci. 2006;29:449-476.

14. Gonçalves SI, de Munck JC, Pouwels PJ, et al. Correlating the alpha rhythm to BOLD using simultaneous EEG/fMRI: inter-subject variability. Neuroimage. 2006;30(1):203-213.

15. Laufs H, Krakow K, Sterzer P, et al. Electroencephalographic signatures of attentional and cognitive default modes in spontaneous brain activity fluctuations at rest. Proc Natl Acad Sci U S A. 2003;100(19):11053-11058.

16. Yang H, Long XY, Yang Y, et al. Amplitude of low frequency fluctuation within visual areas revealed by resting-state functional MRI. Neuroimage. 2007;36(1):144-152.

17. Zang YF, He Y, Zhu CZ, et al. Altered baseline brain activity in children with ADHD revealed by resting state functional MRI. Brain Dev. 2007;29(2):83-91.

18. Hou J, Wu W, Lin Y, et al. Localization of cerebral functional deficits in patients with obsessive-compulsive disorder: a resting-state fMRI study. J Affect Disord. 2012;138(3):313-321.

19. Jiao Q, Ding J, Lu G, et al. Increased activity imbalance in frontosubcortical circuits in adolescents with major depression. PLoS One. 2011;6(9):e25159. 
20. American Psychiatric Association. Diagnostic and Statistical Manual of Mental Disorders, Fourth Edition, Text Revision. Washington, DC: American Psychiatric Association; 2000.

21. Kaufman J, Birmaher B, Brent D, et al. Schedule for Affective Disorders and Schizophrenia for School-Age Children-Present and Lifetime Version (K-SADS-PL): initial reliability and validity data. J Am Acad Child Adolesc Psychiatry. 1997;36(7):980-988.

22. Wood A, Kroll L, Moore A, Harrington R. Properties of the mood and feelings questionnaire in adolescent psychiatric outpatients: a research note. J Child Psychol Psychiatry. 1995;36(2):327-334.

23. Rich BA, Fromm SJ, Berghorst LH, et al. Neural connectivity in children with bipolar disorder: impairment in the face emotion processing circuit. J Child Psychol Psychiatry. 2008;49(1):88-96.

24. Lowe MJ, Mock BJ, Sorenson JA. Functional connectivity in single and multislice echoplanar imaging using resting-state fluctuations. Neuroimage. 1998;7(2):119-132.

25. Pavuluri MN, Sweeney JA. Integrating functional brain neuroimaging and developmental cognitive neuroscience in child psychiatry research. J Am Acad Child Adolesc Psychiatry. 2008;47(11):1273-1288.

26. Dickstein DP, Gorrostieta C, Ombao H, et al. Fronto-temporal spontaneous resting state functional connectivity in pediatric bipolar disorder. Biol Psychiatry. 2010;68(9):839-846.

27. Xiao Q, Zhong Y, Lu D, et al. Altered regional homogeneity in pediatric bipolar disorder during manic state: a resting-state fMRI study. PLoS One. 2013;8(3):e57978.

28. Wu M, Lu LH, Passarotti AM, et al. Altered affective, executive and sensorimotor resting state networks in patients with pediatric mania. J Psychiatry Neurosci. 2013;38(4):232-240.

29. O'Connell RA, Van Heertum RL, Luck D, et al. Single-photon emission computed tomography of the brain in acute mania and schizophrenia. J Neuroimaging. 1995;5(2):101-104.

30. Blumberg HP, Stern E, Martinez D, et al. Increased anterior cingulate and caudate activity in bipolar mania. Biol Psychiatry. 2000;48(11): 1045-1052.

31. Kim P, Jenkins SE, Connolly ME, et al. Neural correlates of cognitive flexibility in children at risk for bipolar disorder. J Psychiatr Res. 2012;46(1):22-30.

32. Passarotti AM, Sweeney JA, Pavuluri MN. Emotion processing influences working memory circuits in pediatric bipolar disorder and attention-deficit/hyperactivity disorder. J Am Acad Child Adolesc Psychiatry. 2010;49(10):1064-1080.

33. Arnone D, Cavanagh J, Gerber D, Lawrie SM, Ebmeier KP, McIntosh AM. Magnetic resonance imaging studies in bipolar disorder and schizophrenia: meta-analysis. Br J Psychiatry. 2009;195(3): 194-201.
34. Liu IY, Howe M, Garrett A, et al. Striatal volumes in pediatric bipolar patients with and without comorbid ADHD. Psychiatry Res. 2011;194(1):14-20.

35. Caligiuri MP, Brown GG, Meloy MJ, et al. An fMRI study of affective state and medication on cortical and subcortical brain regions during motor performance in bipolar disorder. Psychiatry Res. 2003;123(3): $171-182$.

36. Marie JP, Zittoun R, Sikic BI. Multidrug resistance (mdr1) gene expression in adult acute leukemias: correlations with treatment outcome and in vitro drug sensitivity. Blood. 1991;78(3):586-592.

37. Cavanna AE. The precuneus and consciousness. CNS Spectr. 2007; 12(7):545-552.

38. Sohn MH, Ursu S, Anderson JR, Stenger VA, Carter CS. The role of prefrontal cortex and posterior parietal cortex in task switching. Proc Natl Acad Sci U S A. 2000;97(24):13448-13453.

39. van den Heuvel OA, Groenewegen HJ, Barkhof F, Lazeron RH, van Dyck R, Veltman DJ. Frontostriatal system in planning complexity: a parametric functional magnetic resonance version of Tower of London task. Neuroimage. 2003;18(2):367-374

40. Pavuluri MN, Passarotti AM, Parnes SA, Fitzgerald JM, Sweeney JA. A pharmacological functional magnetic resonance imaging study probing the interface of cognitive and emotional brain systems in pediatric bipolar disorder. J Child Adolesc Psychopharmacol. 2010;20(5):395-406.

41. Pavuluri MN, Passarotti AM, Fitzgerald JM, Wegbreit E, Sweeney JA. Risperidone and divalproex differentially engage the fronto-striatotemporal circuitry in pediatric mania: a pharmacological functional magnetic resonance imaging study. J Am Acad Child Adolesc Psychiatry. 2012;51(2):157-170.

42. Cerullo MA, Adler CM, Lamy M, et al. Differential brain activation during response inhibition in bipolar and attention-deficit hyperactivity disorders. Early Interv Psychiatry. 2009;3(3):189-197.

43. Chakravarthy VS, Joseph D, Bapi RS. What do the basal ganglia do? A modeling perspective. Biol Cybern. 2010;103(3):237-253.

44. Stocco A, Lebiere C, Anderson JR. Conditional routing of information to the cortex: a model of the basal ganglia's role in cognitive coordination. Psychol Rev. 2010;117(2):541-574.

45. Phillips ML, Travis MJ, Fagiolini A, Kupfer DJ. Medication effects in neuroimaging studies of bipolar disorder. Am J Psychiatry. 2008;165(3): 313-320.

46. Cardinal TR, Wazlawik E, Bastos JL, Nakazora LM, Scheunemann L. Standardized phase angle indicates nutritional status in hospitalized preoperative patients. Nutr Res. 2010;30(9):594-600.

47. Forstmann BU, Wolfensteller U, Derrfuss J, et al. When the choice is ours: context and agency. PLoS One. 2008;3(4):e1899.
Neuropsychiatric Disease and Treatment

\section{Publish your work in this journal}

Neuropsychiatric Disease and Treatment is an international, peerreviewed journal of clinical therapeutics and pharmacology focusing on concise rapid reporting of clinical or pre-clinical studies on a range of neuropsychiatric and neurological disorders. This journa is indexed on PubMed Central, the 'PsycINFO' database and CAS

\section{Dovepress}

The manuscript management system is completely online and includes a very quick and fair peer-review system, which is all easy to use. Visit http://www.dovepress.com/testimonials.php to read real quotes from published authors. 